UDC 547.569+547.436

\title{
SYNTHESIS OF NEW AMINOMETHYL AND ORGANYLSULPHIDE DERIVATIVES OF PIPERIDINO-2-HYDROXYPROPANETHIOL-3
}

\author{
V.M.Farzaliyev, Sh.R.Aliyev, R.F.Mammadova, R.M.Babai, Q.M.Quliyeva
}

A.Guliyev Institute of Chemistry of Additives, NAS of Azerbaijan

shah.karaca@mail.ru

Received 05.03.2018

\begin{abstract}
At interaction of hydrogen sulphide with piperidino-2,3-epoxyprorane there is formed piperidino-2hydroxypropanthiol-3. The latter reacts by Mannich with formaldehyde and secondary aliphatic and heterocyclic amines, as well with organic halogenide, forming respectively aminomethyl and organylsulphide derivatives of piperidino-2-hydroxypropanethiol-3. Composition and structure of synthesized compounds are confirmed by elemental analysis and method NMR.
\end{abstract}

Keywords: epychlorohydrine, piperidine, formaldehyde, butyl bromide, hydrogen sulphide, aminomethyl derivatives, organylsulphide derivatives.

It is known that the epoxy compounds formed as a result of the interaction of compounds having an active hydrogen atom with epychlorohydrine and sodium hydroxide under treatment by hydrogen sulphide lead to compounds in which the sulphohydryl group is attached to the terminal carbon atom of the propane chain. Analysis of the literature [1-5] shows that such types of compounds have been relatively not enough investigated. However, such compounds may be synthons for the preparation of novel $\mathrm{N}$ - and $\mathrm{S}$ containing compounds that are widely used as biologically active compounds [6], as well as ad- ditives for lubricating oils [7].

In the beginning, piperidino-2,3-epoxypropane was prepared according to the known method [8]. Then by treatment of piperidino-2,3epoxypropane with hydrogen sulphide, piperidino-2-hydroxypropanthiol-3 (I) was synthesized. By acting on the latter as synthon by formalin and secondary aliphatic, heterocyclic amines, and also organyl halogenides, the corresponding aminomethyl (II $a-d$ ) and organylsulphide (III $a-d$ ) derivatives were obtained. The reactions were carried out according to the following schemes:<smiles>OC(CS)CN1CCC(CS)CC1</smiles>

I<smiles>[Y10]CSCC(O)CN1CCC(C[R16]NO[I+])CC1</smiles>

II $a-d$

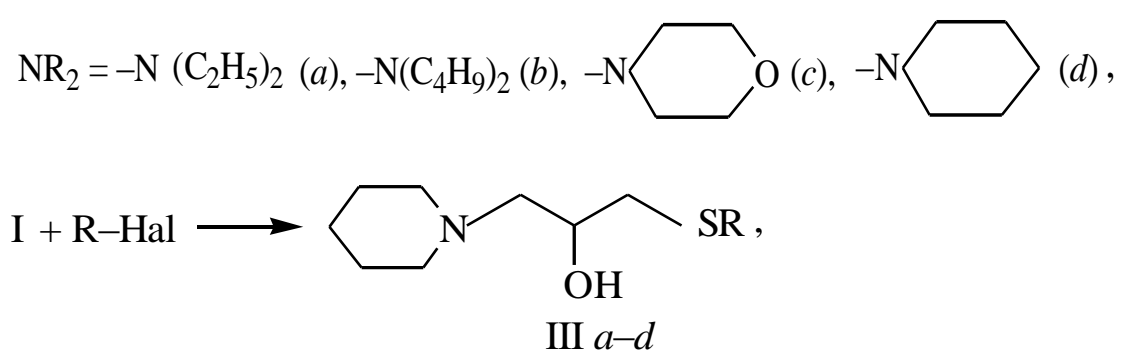

$\mathrm{R}=\mathrm{C}_{4} \mathrm{H}_{9}(a), \mathrm{C}_{7} \mathrm{H}_{15}(b), \mathrm{CH}_{2} \mathrm{COOH}(c), \mathrm{CH}_{2}-\mathrm{CH}_{2} \mathrm{OH}(d)$, Hal = Br, I, Cl. 
Physicochemical characteristics of the obtained compounds are presented in the Table. The composition and structure of the obtained aminomethyl derivatives of piperidino-2-hydroxypropanethiol-3 and organylsulphides are confirmed by elemental analysis and spectral methods.

\section{Experimental part}

NMR spectra of ${ }^{1} \mathrm{H}$ and ${ }^{13} \mathrm{C}$ were taken on a Bruker AV-300, spectrometer at a working frequency of $80 \mathrm{MHz}$. As a solvent $\mathrm{SDCl}_{3}$ was used, the internal standard - hexamethyldisiloxane (HMDS) [9].

Piperidino-2-hydroxypropanethiol-3 (I). $15 \mathrm{~g}$ (0.1 mole) of piperidino-2,3-epoxypropane dissolved in $50 \mathrm{ml}$ of ethyl alcohol and $0.3 \mathrm{~g}$ of $\mathrm{NaOH}$ dissolved in $15 \mathrm{ml}$ of water were placed in a three-necked flask. The reaction mixture was cooled to a temperature of $+5^{0} \mathrm{C}$ and hydrogen sulphide was fed into the mixture for an hour. After completion of the reaction the mixture was extracted by benzene and dried over anhydrous sodium sulphate. After distillation off the solvent, $15.3 \mathrm{~g}(82 \%)$ of compound I was isolated by distillation under vacuum.

Spectrum (compounds I, Figure 1) NMR ${ }^{1} \mathrm{H}, \delta$, ppm: $1.38 \mathrm{tt}\left(2 \mathrm{H},-\mathrm{CH}_{2} \underline{\mathrm{CH}}_{2} \mathrm{CH}_{2}-\right), 1.49 \mathrm{tt}$ $\left(4 \mathrm{H},-\underline{\mathrm{CH}}_{2} \mathrm{CH}_{2} \underline{\mathrm{CH}}_{2}-\right), 2.27 \mathrm{~d}\left(2 \mathrm{H},-\underline{\mathrm{CH}}_{2}-\mathrm{SH}\right), 2.30$ $\mathrm{d}\left(2 \mathrm{H},>\mathrm{NCH}_{2}-\stackrel{\mathrm{OH}}{\mathrm{CH}}-\right), 2.51 \mathrm{tt}\left(4 \mathrm{H},-\underline{\mathrm{CH}_{2}} \underline{\mathrm{NCH}}_{2}-\right)$,

$2.76 \mathrm{~s}(1 \mathrm{H}, \mathrm{SH}), 3.71 \mathrm{~m}(1 \mathrm{H}, \mathrm{CH})$.

${ }^{13} \mathrm{C}$ NMR spectrum, $\delta$, ppm: 24.14 $\left(-\mathrm{CH}_{2} \underline{\mathrm{CH}}_{2} \mathrm{CH}_{2}-\right), 25.97\left(-\underline{\mathrm{CH}}_{2} \mathrm{CH}_{2} \underline{\mathrm{CH}}_{2}-\right), 29.25$

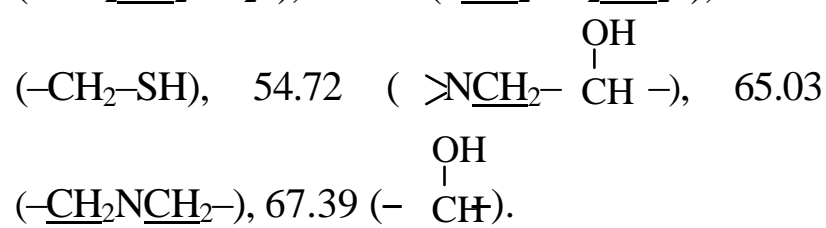

Piperidino-2-hydroxypropyl-3-piperidylmethyl sulphide (II $\boldsymbol{d}$ ). To $6.2 \mathrm{ml}$ of a $20 \%$ aqueous solution of formaldehyde, there were added dropwise at $20-25^{\circ} \mathrm{C} 4 \mathrm{~g}(0.04 \mathrm{~mol})$ of piperidine, then $6 \mathrm{~g}(0.04 \mathrm{~mol})$ of piperidino-2hydroxypropanthiol-3 (I) in $50 \mathrm{ml}$ of benzene. In this cose increase in temperature from 25 to $35^{\circ} \mathrm{C}$ was observed, the mixture was stirred for 4 hours at a temperature of $75-80^{\circ} \mathrm{C}$. After completion of the reaction $28 \%$ sodium hydroxide solution ( $1 \mathrm{~g}$
$\mathrm{NaOH}+2.5 \mathrm{ml} \mathrm{H}_{2} \mathrm{O}$ ) was added to the mixture. Then the benzene solution was washed twice with water and dried with sodium sulphate, then after distillation of benzene the non-distilled liquid (II $d$ ) was extracted.

Compounds (II $a-c$ ) were similarly obtained, the characteristics of which are given in the Table.

Spectrum (compound II $d$, Figure 2) NMR ${ }^{1} \mathrm{H}, \delta$, ppm: $1.44 \mathrm{tt}\left(4 \mathrm{H},-\mathrm{CH}_{2} \underline{\mathrm{CH}_{2}} \mathrm{CH}_{2}-\right), 1.55 \mathrm{tt}$ $\left(8 \mathrm{H},-\underline{\mathrm{CH}_{2}} \mathrm{CH}_{2} \underline{\mathrm{CH}}_{2}-\right), 2.35 \mathrm{~d}\left(2 \mathrm{H},-\underline{\mathrm{CH}}_{2}-\mathrm{S}-\right)$, $\mathrm{OH}$

$2.40 \mathrm{~d} \quad\left(2 \mathrm{H},>\mathrm{NCH}_{2}-\mathrm{CH}-\right), 2.56 \mathrm{tt}(8 \mathrm{H}$, $\left.-\underline{\mathrm{CH}}_{2} \mathrm{NCH}_{2}-\right), 3.51 \mathrm{~s}\left(2 \mathrm{H},-\mathrm{SCH}_{2}-\mathrm{N}<\right), 3.78 \mathrm{~m}$ $(1 \mathrm{H}, \mathrm{CH})$.

${ }^{13} \mathrm{C}$ NMR spectrum, $\delta$, ppm: 24.28 $\left(-\mathrm{CH}_{2} \underline{\mathrm{CH}}_{2} \mathrm{CH}_{2}-\right), 25.97\left(-\underline{\mathrm{CH}}_{2} \mathrm{CH}_{2} \underline{\mathrm{CH}}_{2}-\right), 41.11$ $\begin{array}{cccc}\left(-\underline{\mathrm{CH}}_{2}-\mathrm{S}-\right), & 52.64 \quad\left(>\mathrm{NCH}_{2}-\mathrm{CH}-\right), & 55.20\end{array}$ $\left(-\underline{\mathrm{CH}}_{2} \mathrm{NCH}_{2}-\right), \quad 64.25 \quad\left(-\mathrm{SCH}_{2}-\mathrm{N}<\right), \quad 68.83$ $\mathrm{OH}$ $(-\stackrel{\mathrm{C}}{\mathrm{C}}-)$.

Piperidino-2-hydroxypropyl-3-butylsulphide (III a). In a $100 \mathrm{ml}$ three-necked flask equipped with a stirrer, reflux condenser and dropping funnel, $6 \mathrm{~g}(0.03 \mathrm{~mol})$ of piperidino-2hydroxypropanethiol-3 (I), $30 \mathrm{ml}$ of isopropyl alcohol and $3.6 \mathrm{~g}(0.03 \mathrm{~mol})$ of butyl bromide were placed. While stirring at room temperature, a $32 \%$ solution of $\mathrm{NaOH}(1.04 \mathrm{~g} \mathrm{NaOH}+$ $2.2 \mathrm{ml}$ of $\mathrm{H}_{2} \mathrm{O}$ ) was added dropwise to the mixture, and then the mixture was stirred at 75$80^{\circ} \mathrm{C}$ for 6 hours. After completion of the reaction, the product was extracted by benzene, washed with water, dried over $\mathrm{Na}_{2} \mathrm{SO}_{4}$. After distillation the solvents, the residue was distilled in vacuum.

Compounds (III $b-d)$ were singularly synthesized. Their characteristics listed in the table.

Spectrum (compound III $a$, Figure 3) NMR

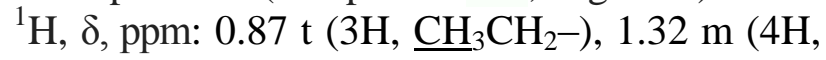
$\left.-\mathrm{CH}_{2} \underline{\mathrm{CH}}_{2} \mathrm{CH}_{3}\right), 1.37$ tt $\left(2 \mathrm{H},-\mathrm{CH}_{2} \mathrm{CH}_{2} \mathrm{CH}_{2}-\right)$; 1.48 tt $\left(4 \mathrm{H},-\mathrm{CH}_{2} \mathrm{CH}_{2} \mathrm{CH}_{2}-\right), 2.24 \mathrm{t}(2 \mathrm{H}$, $\left.-\mathrm{S}-\underline{\mathrm{CH}}_{2}-\mathrm{CH}_{2}-\right), 2.35 \mathrm{~d}\left(2 \mathrm{H},-\underline{\mathrm{CH}}_{2}-\mathrm{S}-\right), 2.39 \mathrm{~d}$ $\mathrm{OH}$

$\left(2 \mathrm{H},>\mathrm{NCH}_{2}-\stackrel{1}{\mathrm{CH}}\right), 2.52 \mathrm{dd}\left(-\underline{\mathrm{CH}}_{2} \underline{\mathrm{NCH}}_{2}-\right)$, $3.75 \mathrm{~m}(1 \mathrm{H}, \mathrm{CH})$. 


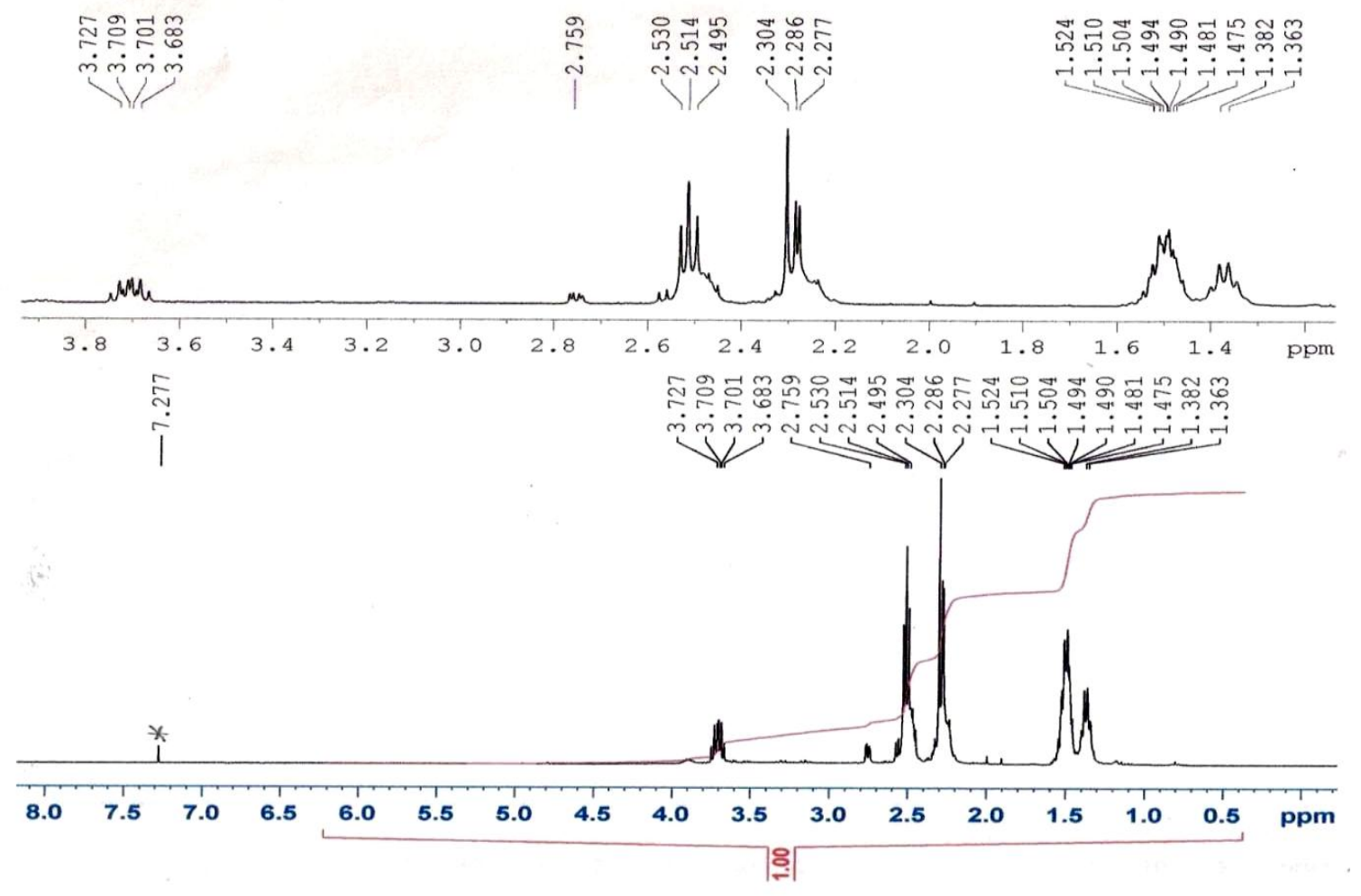

Fig.1. Spectrum (compound I ) NMR ${ }^{1} \mathrm{H}$ piperidino-2-hydroxypropanethiol-3.

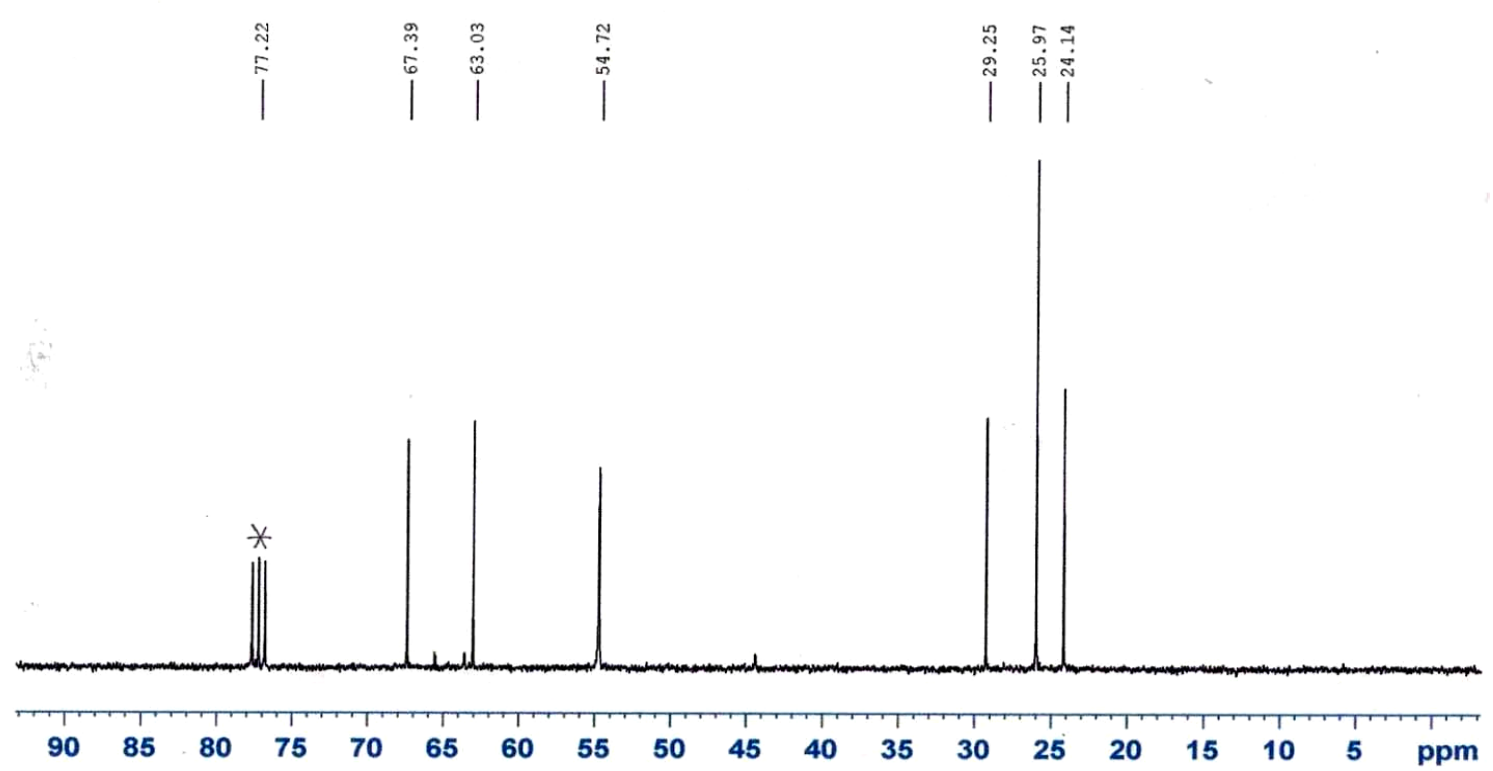

Fig.1. ${ }^{13} \mathrm{C}$ NMR spectrum piperidino-2-hydroxypropanethiol-3 (I). 


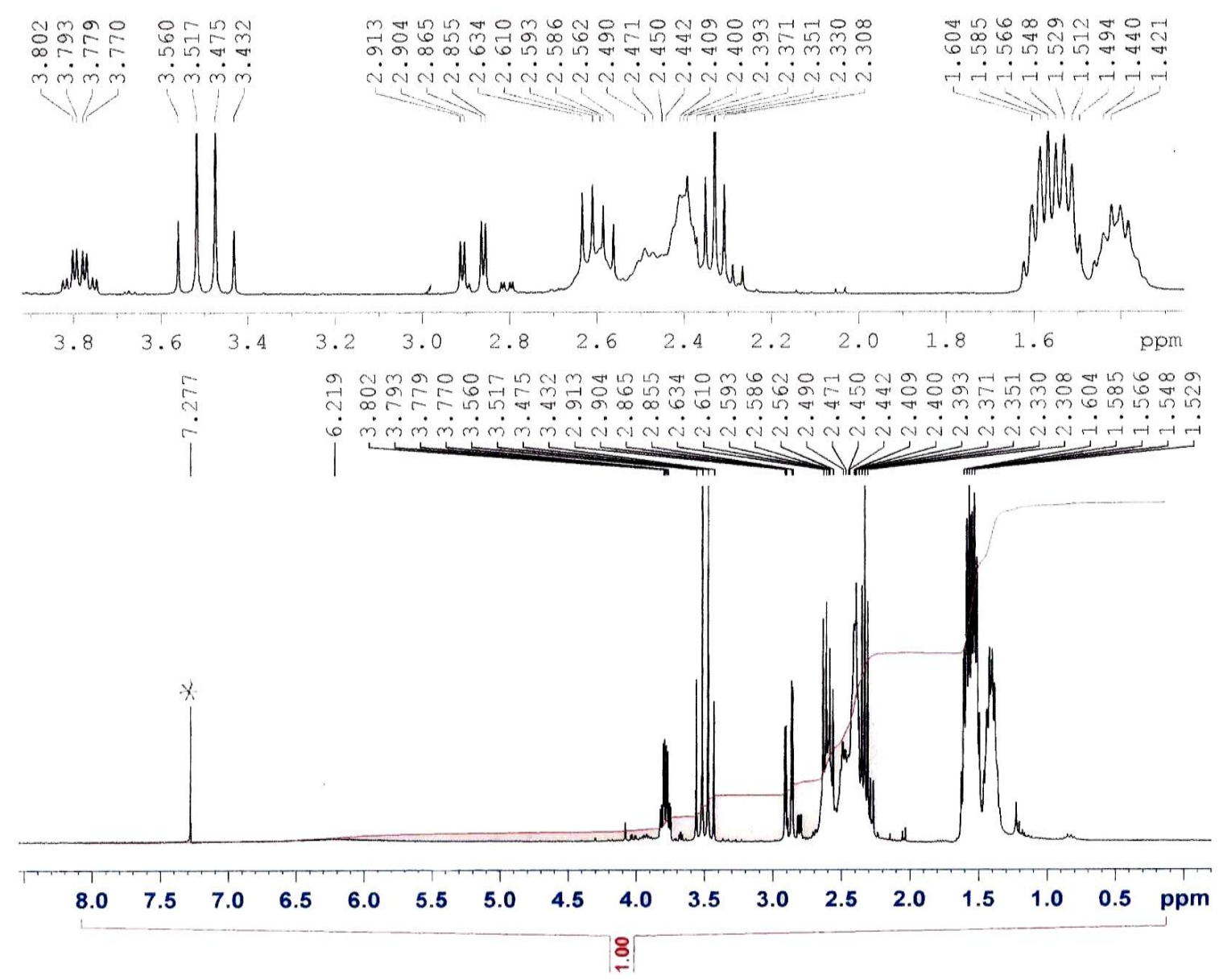

Fig.2. ${ }^{13} \mathrm{C}$ NMR spectrum piperidino-2-hydroxypropyl-3-piperidylmethyl sulfide (IId).

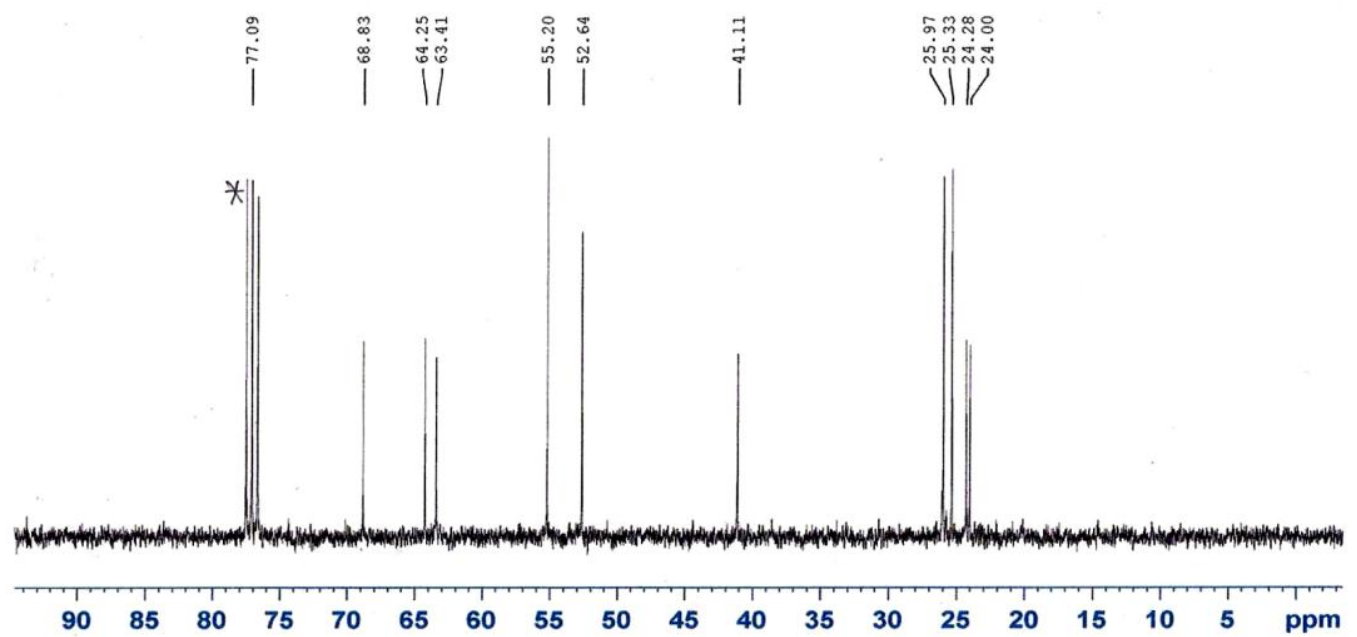

Fig.2. Spectrum (compound IId ) NMR ${ }^{1} \mathrm{H}$ piperidino-2-hydroxypropyl-3-piperidylmethyl sulfide. 


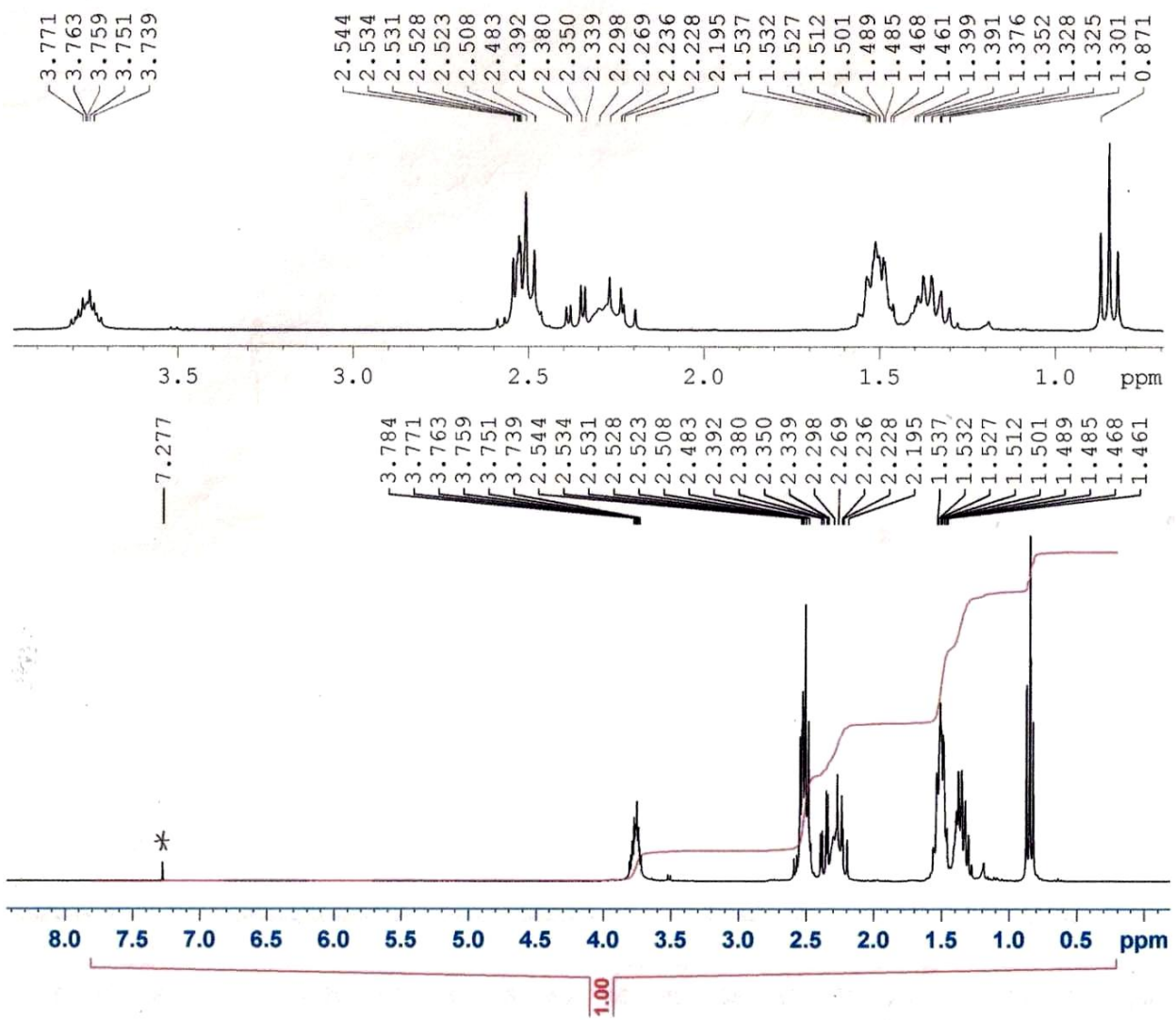

Fig.3. Spectrum (compound III $a$ ) NMR ${ }^{1} \mathrm{H}$ piperidino-2-hydroxypropyl-3-butylsulfide.

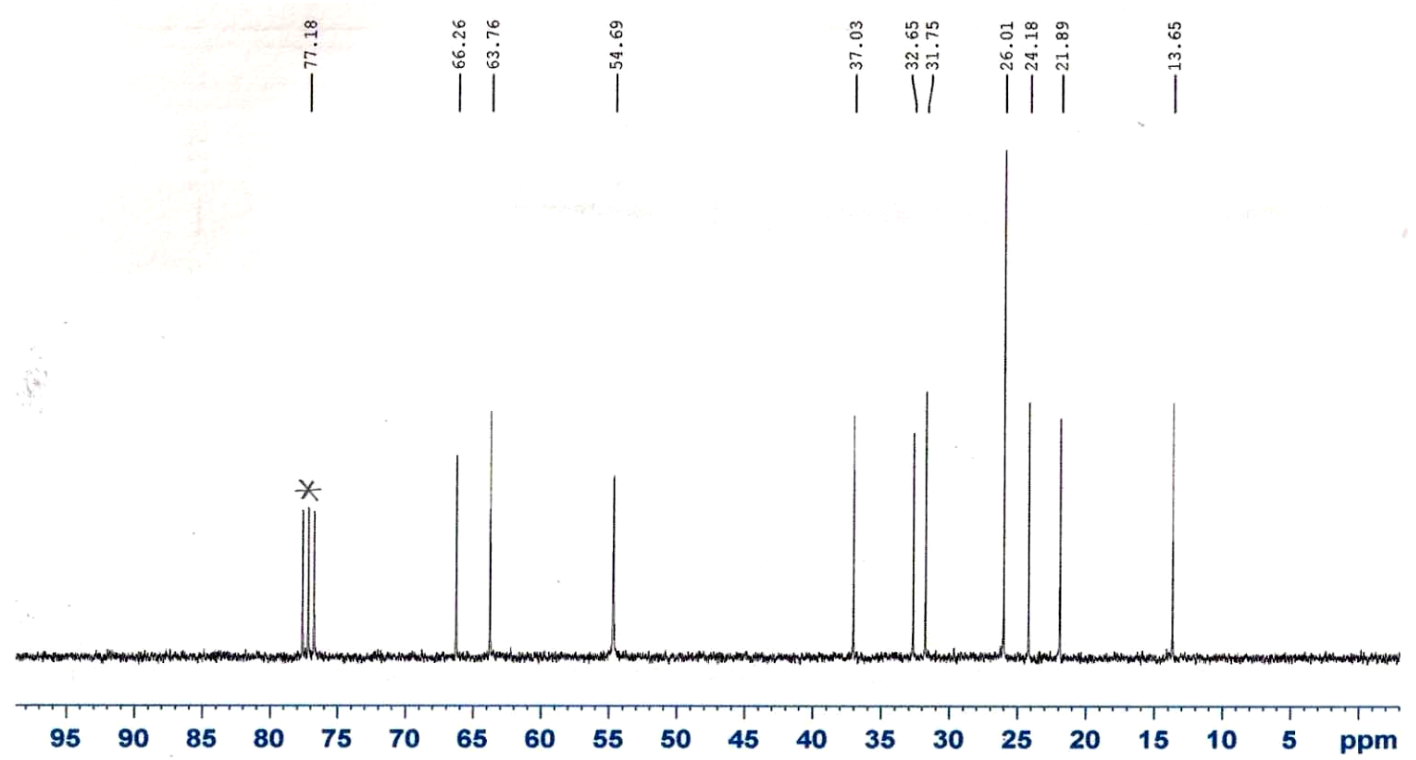

Fig.3. Spectrum (compound III $a$ ) NMR ${ }^{1} \mathrm{H}$ piperidino-2-hydroxypropyl-3-butylsulfide. 
${ }^{13} \mathrm{C} \quad \mathrm{NMR}$ spectrum, $\delta$, ppm: 13.65 $\left(\mathrm{CH}_{3} \mathrm{CH}-\right), \quad 21.89 \quad\left(\mathrm{CH}_{3} \mathrm{CH}_{2}-\right), \quad 24.18$ $\left(-\mathrm{CH}_{2} \underline{\mathrm{CH}}_{2} \mathrm{CH}-\right), 26.01\left(\underline{\mathrm{CH}}_{2} \mathrm{CH}_{2} \underline{\mathrm{CH}}_{2}-\right), 31.75$ $\left(-\mathrm{CH}_{3} \mathrm{CH}_{2} \underline{\mathrm{CH}}_{2}-\right), 32.65\left(2 \mathrm{H},-\mathrm{S}-\underline{\mathrm{CH}}_{2}-\mathrm{CH}_{2}-\right)$,

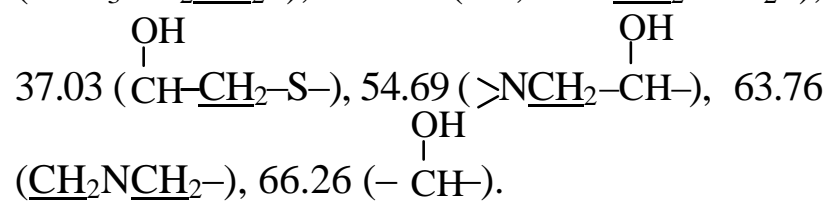

Piperidino-2-hydroxypropyl-3-carboxymethylsulphide (IIIc). In a $100 \mathrm{ml}$ threenecked flask equipped with a stirrer, reflux condenser and dropping funnel, there were placed $4.3 \mathrm{~g}(0.05 \mathrm{~mol})$ of morochloroacetic acid neutralized with a $4 \%$ solution of $\mathrm{NaOH}$ $\left(1.8 \mathrm{~g} \mathrm{NaOH}+44 \mathrm{ml} \mathrm{H}_{2} \mathrm{O}\right), 30 \mathrm{ml}$ of isopropyl alcohol and $8 \mathrm{~g}$ (0.05 mole) piperidino-2hydroxypropanethiol-3 (I) and stirred at room temperature. $32 \%$ solution of $\mathrm{NaOH}$ (1.6 g $\mathrm{NaOH}+3.5 \mathrm{ml} \mathrm{H}_{2} \mathrm{O}$ ) to the reaction mixture was added dropwise. The mixture was then stirred at $70-75^{\circ} \mathrm{C}$ for 6 hours. The obtained reaction mass was hydrolyzed with $0.5 \mathrm{~g}$ of hydrochloric acid. After completion of the reaction, the product was extracted with benzene, washed with water, after distillation of benzene non-distillated liquid (IIIc) was recovered. The characteristic is given in the Table.

\section{References}

1. Farzaliyev V.M., Aliyev Ş.R., Babayi R.M., Guliyeva G.M. Synthesis and study of new protective additives based on secondary heterocyclic amines // J. Gafqaz University Chemistry and Biology. 2016. V. 4. No 1. P. 16-20.

2. Urinov U.K., Maksumova O.S. Studying of the Complex Compounds, formed by molecules of morpholine betaine and urea // Austrian J. Technical and Natural Sciences-Vienna. 2015. No 3-4. P. 70-75.

3. Urinov U.K., Maksumova O.S., Abdulmalikova Kh.B. O reaktcii vzaimodeistviia mocheviny $s$ pikhlorgidrinom // Austrian J. Technical and Natural Sciences-Vienna. 2016. № 3-4. P. 141-145.

4. Sharifova S.K. O napravleniiakh reaktcii epikhlorgidrina s kislotami, spirtami i aminami // Azerb. him. zhurn. 2010. № 2. S. 139-146.

5. Bespalko Iu.N., Shved E.N., Oleinik N.M. Osobennosti kataliticheskogo povedeniia osnovanii v reaktcii alifaticheskikh karbonovykh kislot s $\alpha$-okisiu. // Teor. i eksper. himiia. 2008.T. 44. Vyp. 5. S. 292-297

6. Mashkovskii M.D. Lekarstvennye sredstva. M. Novaia volna, 2002. T. 1, 2. 736; $688 \mathrm{~s}$.

7. Latiuk V.I., Kelarev V.I., Koshelev V.N., Korenev K.D. Sulfidy riada sim-triazina kak maslorastvorimye ingibitory korrozii // Himiia i tekhnologiia topliv i masel. 2002. № 5. S. 23-26.

8. Zeinalov S.B., Kiazimova T.N., Sharifova S.K. Epikhlorgidrin. Baku: Elm, 2000. 188 s.

9. Balci M. Nuclear Magnetic Resonance Spektroscopy. METU Press. Ankara. 2000. 458 p.

\section{PIPERIDIN-2-HIDROKSIPROPANTIOL-3-ÜN YENI AMINOMETIL Və ORQANILSULFID TÖRӘMӘLӘRININ SINTEZI}

\section{V.M.Fərzəliyev, Ş.R.Oliyev, R.F.Məmmədova, R.M.Babayi, Q.M.Quliyeva}

Piperidin və epixlorhidrin əsasında alınmış piperidin-2,3-epoksipropanın qələvi mühitində hidrogen sulfidlə qarşılıqlı təsirindən piperidin-2-hidroksiprorantiol-3 alınmışdır. Sonuncudan sinton maddə kimi istifadə etməklə Mannix reaksiyası şəraitində formaldehid və ikili alifatik, heterotsiklik aminlərlə kondensləşməsindən bir sıra aminometil və orqanil halogenidlərlə reaksiyalarından isə bir sıra orqanilsulfid törəmələri sintez edilmişdir. Alınmış birləşmələrin quruluşu və tərkibi NMR spektroskopiya üsulu və element analizi ilə təsdiq edilmişdir.

Açar sözlor: epixlorhidrin, piperidin, formaldehid, butilbromid, hidrogen sulfid, aminometil töraməlari, orqanilsulfid töramalari.

\section{СИНТЕЗ НОВЫХ АМИНОМЕТИЛЬНЫХ И ОРГАНИЛСУЛЬФИДНЫХ ПРОИЗВОДНЫХ ПИПЕРИДИНО-2-ГИДРОКСИПРОПАНТИОЛ-3}

\section{В.М.Фарзалиев, Ш.Р.Алиев, Р.Ф.Мамедова, Р.М.Бабаи, Г.М.Кулиева}

При взаимодействии в щелочной среде пиперидин-2,3-эпоксипропана, полученного на основе пиперидина и эпихлоргидрина, с сероводородом, синтезирован пиперидин-2-гидроксипропантиол-3. Пользуясь последним как синтоном, в условиях реакции Манниха при конденсации формальдегида и вторичных алифатических, гетероциклических аминов получен ряд аминометильных, а реакциями с органилгалогенидами - ряд органилсульфидных производных. Состав и структура синтезированных соединений подтверждены данными элементного анализа и методом ЯМР.

Ключевые слова: эпихлоргидрин, пиперидин, формальдегид, бромистылй бутил, сероводород, аминометильные производные, огранилсульфидные производнье. 
The yields, constants and elemental analysis data of piperidino-2-hydroxypropanethiol-3 (I) and its aminomethyl (II $a-d$ ) and organylsulphide derivatives (III $a-d$ )

\begin{tabular}{|c|c|c|c|c|c|c|c|c|c|c|}
\hline \multirow{2}{*}{$\begin{array}{l}\text { № com- } \\
\text { pounds }\end{array}$} & \multirow{2}{*}{$\begin{array}{c}\text { Yield, } \\
\%\end{array}$} & \multirow{2}{*}{$\begin{array}{l}T_{\text {boil }}, \\
{ }^{0} \mathrm{C}\end{array}$} & \multirow{2}{*}{$d_{4}^{20}$} & \multirow{2}{*}{$n_{D}^{20}$} & \multicolumn{4}{|c|}{ Found/calculated, $\%$} & \multirow{2}{*}{$\begin{array}{l}\text { Brutto } \\
\text { formula }\end{array}$} & \multirow{2}{*}{$\begin{array}{c}M R_{D} \\
\text { Found/calculated, \% }\end{array}$} \\
\hline & & & & & $\mathrm{C}$ & $\mathrm{H}$ & $\mathrm{N}$ & $\mathrm{S}$ & & \\
\hline I & 82 & $\begin{array}{c}115-117 \\
(3 \mathrm{~mm})\end{array}$ & 1.0321 & 1.4978 & $\frac{54.82}{55.24}$ & $\frac{9.78}{9.42}$ & $\frac{7.99}{8.47}$ & $\frac{18.29}{18.70}$ & $\mathrm{C}_{8} \mathrm{H}_{17} \mathrm{NOS}$ & $\frac{49.77}{50.22}$ \\
\hline II $a$ & 78 & $\begin{array}{l}\text { Non-permeating } \\
\text { clear liquid }\end{array}$ & 0.9552 & 1.4768 & $\underline{59.95}$ & $\underline{10.84}$ & $\frac{10.76}{10.45}$ & $\frac{12.31}{12.70}$ & $\mathrm{C}_{13} \mathrm{H}_{28} \mathrm{~N}_{2} \mathrm{OS}$ & $\frac{77.00}{77.36}$ \\
\hline II $b$ & 68 & " --_-- " & 0.9466 & 1.4892 & $\frac{64.50}{65.00}$ & $\frac{11.46}{11.05}$ & $\frac{8.85}{8.54}$ & $\frac{10.13}{9.84}$ & $\mathrm{C}_{17} \mathrm{H}_{36} \mathrm{~N}_{2} \mathrm{OS}$ & $\frac{96.55}{96.03}$ \\
\hline II $c$ & 80 & " -ב-_" & 1.0632 & 1.5117 & $\frac{57.40}{56.90}$ & $\frac{9.13}{9.55}$ & $\frac{9.81}{10.21}$ & $\frac{12.13}{11.68}$ & $\mathrm{C}_{13} \mathrm{H}_{26} \mathrm{~N}_{2} \mathrm{O}_{2} \mathrm{~S}$ & $\frac{77.41}{77.00}$ \\
\hline II $d$ & 85 & " --_- " & 1.0364 & 1.5220 & $\frac{61.72}{62.02}$ & $\frac{10.36}{10.15}$ & $\frac{10.28}{10.59}$ & $\frac{11.77}{12.18}$ & $\mathrm{C}_{14} \mathrm{H}_{28} \mathrm{~N}_{2} \mathrm{OS}$ & $\frac{80.24}{79.97}$ \\
\hline III $a$ & 85 & $\begin{array}{c}134-135 \\
(2 \mathrm{~mm})\end{array}$ & 0.9921 & 1.4953 & $\frac{62.29}{61.93}$ & $\frac{10.89}{10.61}$ & $\frac{6.05}{6.41}$ & $\frac{13.86}{13.47}$ & $\mathrm{C}_{12} \mathrm{H}_{25} \mathrm{NOS}$ & $\frac{68.05}{68.81}$ \\
\hline III $b$ & 88 & $\begin{array}{c}162-163 \\
(1.5 \mathrm{~mm})\end{array}$ & 0.9516 & 1.4878 & $\frac{65.88}{65.37}$ & $\frac{11.43}{11.92}$ & $\frac{5.42}{4.96}$ & $\frac{11.72}{11.41}$ & $\mathrm{C}_{15} \mathrm{H}_{31} \mathrm{NOS}$ & $\frac{82.77}{82.66}$ \\
\hline III $c$ & 87 & $\begin{array}{l}\text { Non-permeating } \\
\text { clear liquid }\end{array}$ & 1.1254 & 1.4932 & $\frac{51.48}{51.00}$ & $\frac{8.21}{8.49}$ & $\frac{6.00}{6.28}$ & $\frac{13.74}{14.14}$ & $\mathrm{C}_{10} \mathrm{H}_{19} \mathrm{NO}_{3} \mathrm{~S}$ & $\frac{60.27}{60.66}$ \\
\hline III $d$ & 85 & " & 1.1167 & 1.5302 & $\frac{55.27}{54.84}$ & $\frac{8.81}{9.03}$ & $\frac{6.45}{6.83}$ & $\frac{14.75}{14.28}$ & $\mathrm{C}_{10} \mathrm{H}_{21} \mathrm{NO}_{2} \mathrm{~S}$ & $\frac{60.69}{61.20}$ \\
\hline
\end{tabular}

